Original Research Article

\title{
Drug utilization pattern in out-patients with respiratory tract infections in a rural teaching hospital: a prospective observational study
}

\author{
Paramita Pal $^{1}$, Dipankar Bhattacharyya ${ }^{2 *}$, Kokila B. N. ${ }^{3}$, Herle M. ${ }^{3}$, \\ Anoljyoti Ghosh ${ }^{4}$, Sukanta Sen ${ }^{1}$
}

\begin{abstract}
${ }^{1}$ Department of Pharmacology,
${ }^{2}$ Department of Physiology,

ICARE Institute of Medical

Sciences and Research,

Banbishnupur, Purba Medinipur, Haldia, West Bengal 721645,

India

${ }^{3}$ Department of Pharmacology,

KVG Medical College \&

Hospital, Sullia, Karnataka

574327, India

${ }^{4}$ Department of Pharmacology,

Mata Gujari Memorial Medical

College, Purabbali, Dinajpur

Road, Kishanganj, Bihar

855108, India
\end{abstract}

Received: 06 October 2017

Revised: 13 October 2017

Accepted: 28 October 2017

\section{*Correspondence to:}

Dr. Dipankar Bhattacharyya, Email: drdbhat1981@gmail.com

Copyright: (C) the author(s), publisher and licensee Medip Academy. This is an openaccess article distributed under the terms of the Creative Commons Attribution NonCommercial License, which permits unrestricted noncommercial use, distribution, and reproduction in any medium, provided the original work is properly cited.

\begin{abstract}
Background: Respiratory tract infections are common clinical problems in the general population. Antimicrobials are the mainstay in the management and irrational use of them may increase resistance to bacteria and the total cost of treatment. Objectives: To evaluate the pattern of drug prescriptions for respiratory tract infections in Medicine and Pediatric outpatient departments of a rural teaching hospital.

Methods: Over a period of 18 months, 391 prescriptions of outpatients with respiratory tract infection were collected. The drugs prescribed, their dose and duration of treatment were recorded. DU $90 \%$ was calculated.

Results: The mean $( \pm$ SEM $)$ age of the patients was38.55 \pm 0.9 years and there were $204(52.1 \%)$ men and $187(42.1 \%)$ women. The most common disorder among the patients was acute rhinitis $(61.1 \%)$ while the least common was acute bronchitis $(1.3 \%)$. Microbial culture and sensitivity was done in 23 patients and Klebsiella pneumonia $(8.2 \%)$ and enterococcus $(0.07 \%)$ was the most common and least common organism respectively. Penicillins $(50.9 \%)$, cephalosporins (26.7\%), antitubercular drugs (8.5\%), macrolides (4.8) constituted DU 90\%. Monotherapy was advocated in $91.7 \%$ and multidrug therapy in $8.3 \%$ of patients. The average number of antimicrobials prescribed per prescription was 0.52 . Two thirds $(67.6 \%)$ of the prescribed drugs were from the national list of essential medicines 2011 (NLEM).

Conclusions: Penicillins and cephalosporins were the commonly used antibiotics for respiratory tract infection in outpatients of a rural teaching hospital and two thirds of the prescribed drugs were essential medicines.
\end{abstract}

Keywords: Antimicrobial agents, Drug utilization, Respiratory tract infections

\section{INTRODUCTION}

Respiratory tract Infections (RTIs) are common clinical problems frequently seen in both children and adults and responsible for considerable morbidity, distress and mortality. Acute respiratory infections account for $20-40 \%$ of outpatient attendance in a general hospital. ${ }^{1,2}$ Upper respiratory tract infections (URTIs) constitute the majority of respiratory tract infections and the presenting manifestations are coughs or colds (coryza). Drug therapy for the symptoms of upper respiratory infections is sought 
for the relief of discomfort and for the alleviation of the anxiety that the URTIs are potentially serious. ${ }^{3}$

Lower respiratory tract infections (LRTIs) can be applied to pneumonia and other types of infection including lung abscess and acute bronchitis. Symptoms include shortness of breath, weakness, high fever, coughing and fatigue. The two most common LRTIs are bronchitis and pneumonia, pneumonia is the fourth leading cause of death. ${ }^{4,5}$ LRTIs impose a considerable cost to the nation. ${ }^{6}$

Antibiotics are often thought to be the first line treatment in lower respiratory tract infections; however, these are not indicated in viral infections where antiviral therapy is required. It is important to use appropriate antibiotic selection based on the infecting organism and to ensure this therapy changes with the evolving nature of these infections and the emerging resistance to conventional therapies. $^{7}$

Majority of prescriptions of antimicrobial agents in community practice are for the treatment of acute upper respiratory tract infections (URTIs), including rhinosinusitis, pharyngitis, bronchitis and nonspecific URTIs, despite the fact that viruses cause most of these illnesses. ${ }^{8}$ This widespread indiscriminate use of antibiotics has contributed to the spread of antibiotic resistance among community-acquired pathogens, such as Streptococcus pneumoniae.

Common lower respiratory tract pathogens have become more resistant to antibiotics. ${ }^{9}$ Penicillin resistant and multidrug-resistant Streptococcus pneumoniae are increasingly common. ${ }^{10,11}$ Case reports have described treatment failures related to quinolone resistance. ${ }^{12,13}$

Prescription of drugs needs to be continuously assessed and refined according to clinical pattern of disease and evolving new organisms with different drug sensitivity. It not only reflects the physician's knowledge about drugs but also his or her skills in diagnosing and attitude towards selecting the most appropriate cost-effective treatment. Antimicrobials are among the most commonly prescribed drugs in hospital. They account for over $50 \%$ of total value of drugs sold in our country. ${ }^{14}$ Antimicrobials are second most commonly used drug in USA. ${ }^{15}$

The inappropriate and economically inefficient use of medication in terms of poly pharmacy, use of drugs not related to the diagnosis, poor patient's compliance, overuse and misuse of antibiotics and use of unnecessary expensive drugs has been commonly observed in the health care system throughout the world, especially in the developing countries. ${ }^{15}$

Unnecessary antibiotic prescription remains common not only in developing countries, but also in developed countries like USA and Britain. ${ }^{7}$ In India, $69.4 \%$ of patients with uncomplicated acute respiratory infections were prescribed antibiotics. ${ }^{16}$ A WHO study of antimicrobial use in 13 low-middle and high-income countries revealed that antimicrobials were wrongly prescribed for approximately $30 \%$ of cases of URTI. ${ }^{17}$

Accordingly, in view of the aforementioned observations, this prospective study envisages compilation, analysis of pattern, trend, rationality, and frequency of the use of drugs in the treatment of respiratory tract infections, with emphasis on available treatment regimens inclusive of primary and adjuvant therapy.

\section{METHODS}

This prospective, non-interventional, observational and medical audit-study was conducted at Department of General Medicine and the Department of Pediatrics at the KVG Medical College, Sullia, Dakshin Kannada District, Karnataka, India. A total sample size of 391 was taken for this study. Study was carried out during the period of January 2013 to June 2014. A pre-designed proforma for data collection was used to collect relevant information from the out-patients' prescription slips. Ethical committee clearance was obtained for the conduct of the study.

\section{Inclusion criteria}

Patients above five years of age of either gender suffering from respiratory tract infections.

\section{Exclusion criteria}

- Patients below five years.

- Pregnant mothers with RTIs.

Demographic data and clinical condition for which the drugs were prescribed were captured. Details of the drugs prescribed including drugs name, class, dosage, route of administration, likely duration of treatment was also documented. The data collected in a specially designed proforma were processed and subjected to relevant statistical analysis. Descriptive statistical procedure and evaluation were done to analyze the results using SPSS for windows (version19). Descriptive statistical analysis of the data was done in the form of pie-chart and bar diagrams.

\section{RESULTS}

A total number of 800 prescriptions were screened over a study period of 18 months from January 2013 to June 2014 in two departments (Medicine and Pediatrics) and out of which 391 prescriptions were collected, scrutinized and analyzed for epidemiologic profile, disease incidence and drug prescription. Accordingly, the results of this observational, prospective and medical audit study are as follows:

In this study patients of either gender and above 5years of age have been selected for the scrutiny of the prescription 
patterns. The respective tables 1-15 indicate the overall details regarding the respiratory tract infections.

Table 1 illustrate that the total number of male patients affected with respiratory tract infections $(54.49 \%)$ was more than the female patients $(45.51 \%)$. Also incidence of URTI in men $(49.37 \%)$ and women $(42.19 \%)$ were more compared to LRTI (male-5.12\%, female-3.32\%).

Table 1: Pattern of respiratory tract infections in the study participants $(n=391)$.

\begin{tabular}{|lllllll|}
\hline & Male & \multicolumn{3}{c|}{ Female } & Total \\
\hline Disease & No. & $\%$ & No. & $\%$ & No. & $\%$ \\
\hline URTI & 193 & $49.37 \%$ & 165 & $42.19 \%$ & 358 & $91.56 \%$ \\
\hline LRTI & 20 & $5.12 \%$ & 13 & $3.32 \%$ & 33 & $8.44 \%$ \\
\hline Total & 213 & $54.49 \%$ & 178 & $45.51 \%$ & 391 & $100 \%$ \\
\hline
\end{tabular}

Table 2 indicates the incidence of the various infective conditions of respiratory tract in different age groups. Most of the URTIs recorded in this study have shown a predilection for the age group 5 to 25 years (40.79\%) followed by the age group 26 to 50 years $(38.23 \%)$ with a slight variation, whereas, most of the LRTIs which have been studied show predilection for the age group 51 to 75 years $(4.01 \%)$.

Table 2: Age prevalence of patients in RTIs $(n=391)$.

\begin{tabular}{|lllll|}
\hline Age in years & URTI & $\%$ & LRTI & $\%$ \\
\hline $5-25$ & 160 & $40.79 \%$ & 9 & $2.31 \%$ \\
\hline $26-50$ & 149 & $38.23 \%$ & 4 & $1.02 \%$ \\
\hline $51-75$ & 37 & $9.45 \%$ & 16 & $4.01 \%$ \\
\hline$>75$ & 12 & $3.09 \%$ & 4 & $1.10 \%$ \\
\hline
\end{tabular}

Figure 1 highlights the distribution of various types of respiratory tract infections in terms of numbers and percentage as per the medical case records of this study.

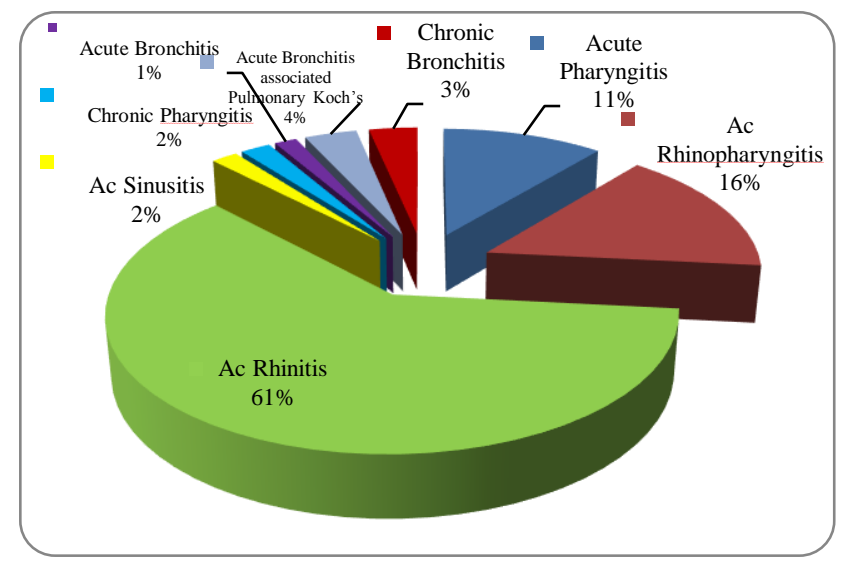

Figure 1: Distribution of RTIs.

Accordingly, acute rhinitis $(61 \%)$ appears to be the most prevalent respiratory tract infective condition followed by acute rhinopharyngitis (16\%), acute pharyngitis $(11 \%)$, pneumonia $(6.13 \%)$, acute bronchitis with pulmonary
Koch's (3.15\%), chronic bronchitis (3\%), chronic pharyngitis $(2 \%)$, acute sinusitis $(2 \%)$, acute bronchitis $(1 \%)$ in that decreasing order.

Furthermore, regarding the laboratory investigations, in 85 out of 391 case records, relevant materials were sent for culture and sensitivity tests. Out of 85 cases sent for culture and sensitivity tests, pathogenic organisms were found in 19 cases, while no bacterial isolates were observed in the remaining 66 cases (Figure 2). Among the 43 cases where bacterial isolates were found Klebsiella pneumoniae (8.32\%), Pseudomonas aeruginosa (3.53\%), Escherichia coli $(2.35 \%)$, Streptococcus pneumoniae (1.18\%) and Enterococcus spp. (1.18\%) were the common pathogens noted (Figure 2).

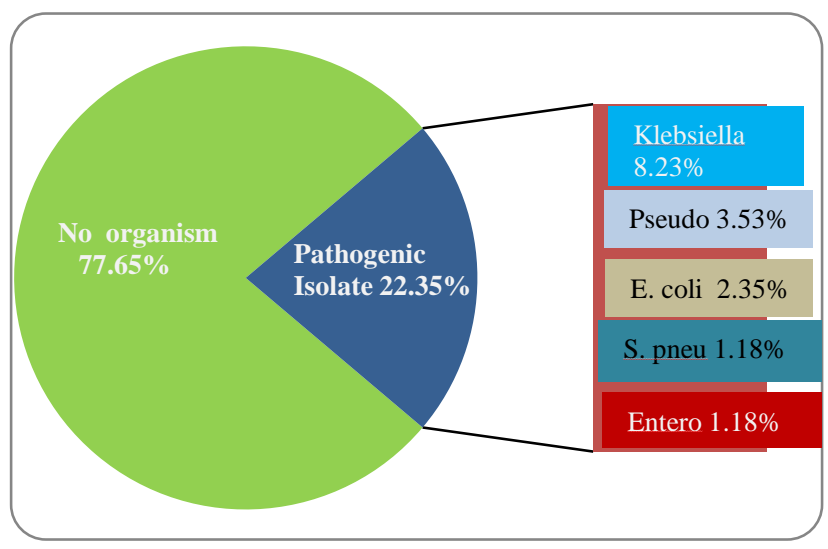

Figure 2: Distribution of organisms in RTIs.

\section{Drug utilization data in RTIs}

On scrutiny, the prescription patterns reveal the use of antimicrobial agents, bronchodilators, $\mathrm{H}_{1}$ antihistamines, expectorants, mucolytics, cough suppressants, decongestants, gastroprotective agents $\left(\mathrm{H}_{2}\right.$ antihistamines and proton pump inhibitors), and leukotriene antagonists as important therapeutic remedies in the respective respiratory tract infective disorders. Figure 3 illustrates the various combinations in regard to monotherapy (169) and polytherapy (37) regimens in various conditions of respiratory tract infections.

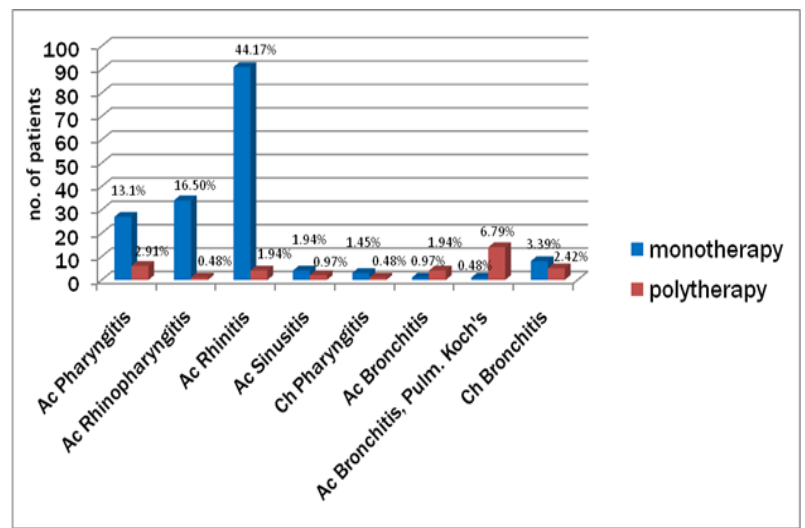

Figure 3: Monotherapy and polytherapy in RTIs. 
A total number of 1236 drugs were prescribed out of it, number of antimicrobials prescribed was 352 and adjuvants were 884 . Figure 4 enlighten the extent and pattern of overall group wise AMAs prescriptions in total sample of 391 patients. Specific antimicrobials used include the antibacterials viz. penicillins (50.85\%) (amoxicillin, amoxicillin-clavulinic acid combination), cephalosporins (26.70\%) (cefixime, ceftriaxone), aminoglycosides $(1.98 \%)$ (amikacin), macrolides $(4.82 \%)$ (azithromycin, clarithromycin), fluoroquinolones (3.12\%) (levofloxacin, ciprofloxacin), antitubercular drugs $(8.52 \%)$ (RIM, PZN, INH, ETH).

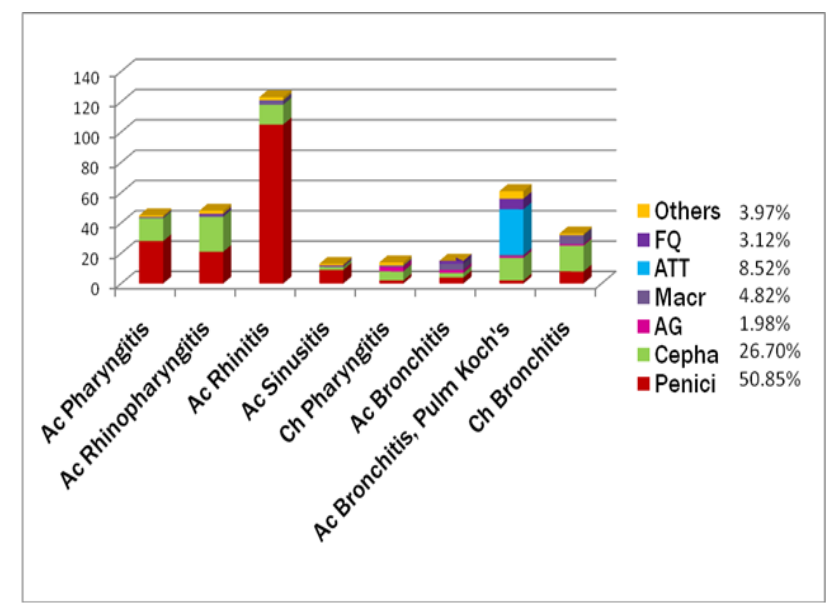

AMAs=Antimicrobial Agents, $\mathrm{FQ}=$ Fluoroquinolone, $\mathrm{ATT}=\mathrm{Anti}$ Tubercular Treatment, Macr=Macrolide, $\mathrm{AG}=\mathrm{Aminoglycoside}$, Cepha=Cephalosporin, Penici=Penicillin

\section{Figure 4: Presentation of AMAs Used in RTIs.}

Figure 5 highlight the important categories of adjuvants co-prescribed with AMAs in relevant clinical situations such as bronchodilators (26.47\%), expectorants, mucolytics, cough suppressants, decongestants (46.04\%), $\mathrm{H}_{1}$ antihistamines $(8.03 \%)$, gastroprotective agents $(8.14 \%)$, antipyretics $(9.95 \%)$, corticosteroids $(1.35 \%)$ and few other drugs.

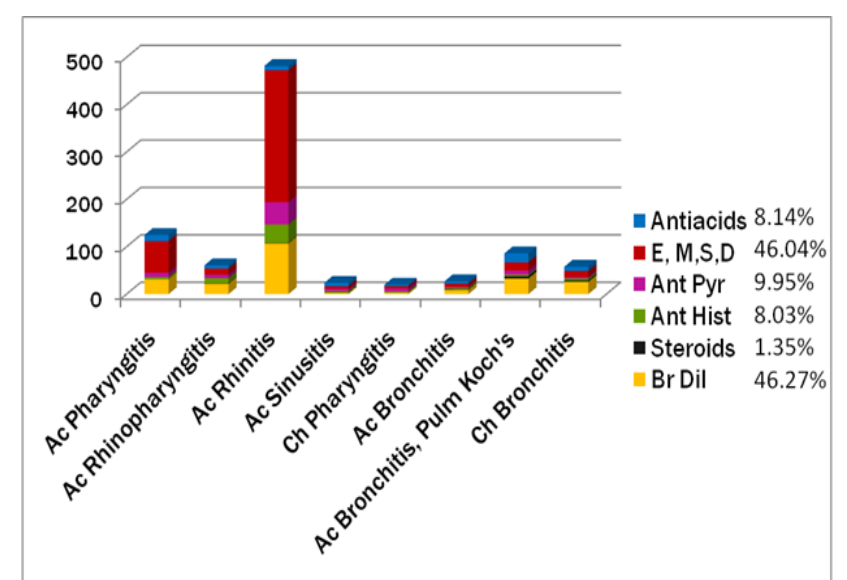

E=Expectorant, $\mathrm{M}=$ Mucolytic, $\mathrm{S}$ =Cough Suppressant, $\mathrm{D}=$ Decongestant, Ant Hist=Antihistamine, Ant $\mathrm{Pyr}=$ Antipyretic, Br Dil=Bronchodilator

Figure 5: Representation of adjuvants used in RTIs.
The chosen bronchodilators used as adjuvants concurrently or separately are salbutamol, salmeterol, albuterol, terbutaline, aminophylline, theophylline, ipratropium bromide. Adjuvant drugs used systematically were chlorpheniramine, cetirizine, levocetirizine, loratadine $\left(\mathrm{H}_{1}\right.$ antihistamine $)$ ranitidine $\left(\mathrm{H}_{2}\right.$ antihistamine), omeprazole, pantoprazole, rabeprazole (proton pump inhibitor).

Corticosteroids employed as the adjuvants were budesonide, dexamethasone, hydrocortisone, beclomethasone and prednisolone.

Expectorants, mucolytics, cough suppressants and decongestants commonly included were bromhexine, guaiphenesin, ambroxol, noscapine, dextromethorphan, phenylephrine. Xylometazoline was the most common decongestant used topically. The chosen NSAIDs used as concurrently or separately are diclofenac, ibuprofen and paracetamol.

Figure 6 show various AMAs constituting DU90\% segment. Here DU90\% segment was mainly constituted by penicillins, cephalosporins, aminoglycosides, macrolides, antitubercular treatment and fluoroquinolones. Out of 12 different AMAs prescribed in this study, 6 drugs were part of DU90\% segment.

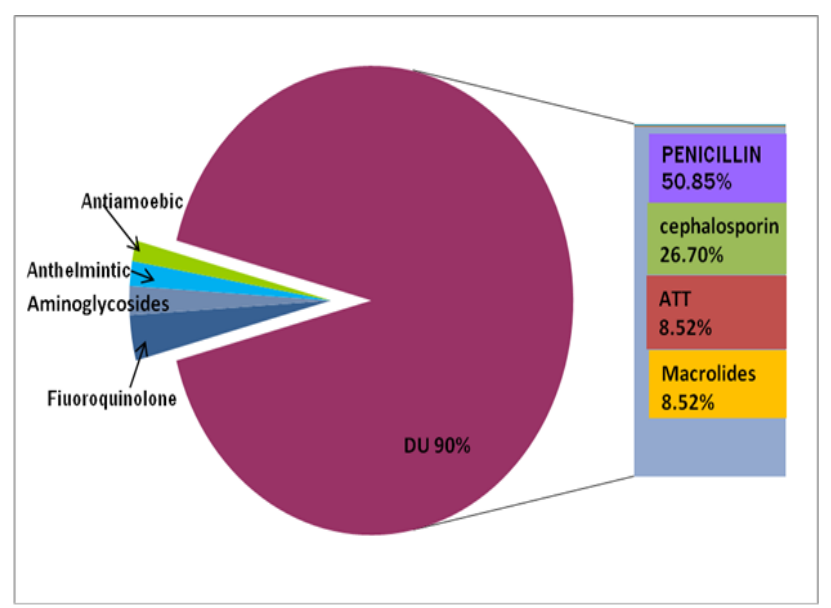

DU-Drug Utilization, ATT- Anti Tubercular Treatment

Figure 6: Illustrations of AMAs constituting DU90\%.

Chi-square test shows no significant difference in the use of AMAs in various infective conditions in out-patient department (Table 3). Most of the outpatients reported were suffering from acute rhinitis and acute rhinosinusitis which are viral in origin and were treated symptomatically without any antimicrobial agents. AMAs were prescribed for patients complaining of symptoms of pharyngitis or other severe symptoms.

Table 4 shows different prescribing parameters obtained from this medical-audit study. This table illustrates that average number of drugs prescribed per prescription was 3.16 and the percentage of drugs prescribed by generic 
name was negligible $(0.56 \%)$. The table also demonstrates that the percentage of prescriptions with antibiotics was $52.68 \%$ which accounts for the fact that most of the RTIs had been treated with antimicrobials. Percentage of prescriptions with injection prescribed was $1.19 \%$ whereas the percentage of drugs prescribed from Essential Drug List 2011 (NLEM 2011) was 67.60\%. It shows that moderate numbers of drugs were prescribed from the essential list and there is still scope for prescribing more drugs from the Essential Drug List (NLEM 2011).

Table 3: Illustration of mean number of AMAs used in RTIs.

\begin{tabular}{|lll|l|}
\hline RTIs & $\begin{array}{l}\text { No. of } \\
\text { cases }\end{array}$ & $\begin{array}{l}\text { No. of } \\
\text { AMAs }\end{array}$ & Mean \pm SE \\
\hline Acute pharyngitis & 57 & 43 & $0.71 \pm 0.09$ \\
\hline Acute rhinitis & 258 & 132 & $0.51 \pm 0.03$ \\
\hline $\begin{array}{l}\text { Acute } \\
\text { rhinopharyngitis }\end{array}$ & 73 & 56 & $0.77 \pm 0.08$ \\
\hline Acute sinusitis & 7 & 13 & $1.83 \pm 0.47$ \\
\hline Chronic pharyngitis & 8 & 14 & $1.71 \pm 0.35$ \\
\hline Acute bronchitis & 30 & 53 & $1.75 \pm 0.15$ \\
\hline $\begin{array}{l}\text { Acute bronchitis, } \\
\text { Acute COPD }\end{array}$ & 82 & 145 & $1.76 \pm 0.11$ \\
\hline $\begin{array}{l}\text { Acute bronchitis, } \\
\text { Pulmonary Koch's }\end{array}$ & 19 & 66 & $3.27 \pm 0.49$ \\
\hline Atypical pneumonia & 3 & 8 & $2.66 \pm 0$ \\
\hline Pneumonia & 37 & 103 & $2.08 \pm 0.19$ \\
\hline Pulmonary Koch's & 16 & 85 & $5.2 \pm 0.39$ \\
\hline Chronic Bronchitis & 13 & 20 & $1.58 \pm 0.22$ \\
\hline Total & 603 & 738 & $1.22 \pm 0.05$ \\
\hline
\end{tabular}

Table 4: Prescription parameters $(n=391)$.

\begin{tabular}{|ll|}
\hline $\begin{array}{l}\text { Average number of drugs per } \\
\text { prescription }\end{array}$ & $\mathbf{3 . 1 6}$ \\
\hline$\%$ of drugs prescribed by generic name & $0.56 \%$ \\
\hline$\%$ of prescription with antibiotic & $52.68 \%$ \\
\hline$\%$ of prescription with injection & $1.19 \%$ \\
\hline$\%$ of drugs from essential drug list & $67.60 \%$ \\
\hline
\end{tabular}

In general, the routes of drug administration in the respiratory tract infections have been oral with a few exceptions wherein, certain antimicrobial agents have also been administered parenterally. Adjuvants have been administered through oral, parenteral and inhalational route.

\section{DISCUSSION}

The study was conducted in two major departments (Medicine and Pediatrics) of K.V.G. Medical College and Hospital, Sullia, over a period of 18 months (from Jan 2013 to Jun 2014) including 2 months of pilot study. Among the 4032 prescriptions screened, 391 medical case records of patients affected with RTIs were analyzed for prescription pattern. The mean age of presentation was observed to be $38.55 \pm 0.9$ years. The percentage of male patients suffering from RTIs in this study was more $(57.87 \%)$ compared to females $(42.12 \%)$ whereas demographic characteristics in similar studies have shown that percentage of females suffering from RTIs was more than males. ${ }^{17,18}$

Acute rhinitis $(61.12 \%)$ appears to be the most common respiratory tract infection and acute bronchitis (1.27\%) was the least common. Out of 85 cases sent for culture and sensitivity tests, pathogenic organisms were found in 19 cases, while no bacterial isolates were observed in the remaining 66 cases. Klebsiella pneumonia $(8.23 \%)$ and enterococcus $(1.18 \%)$ was the most common and least common organism respectively. A total number of 1236 drugs were prescribed out of it, number of antimicrobials prescribed was 352 and adjuvants were 884 . Penicillins (50.9\%), cephalosporins (26.7\%), antituberculardrugs (8.5\%), macrolides (4.8) constituted DU $90 \%$.

In the adjunctive therapy pertaining to RTIs cough suppressants, decongestants, expectorants and mucolytics were the most commonly used drug. Bronchodilators were the other commonly used adjuncts in all cases. No drugs known to cause drug interactions were used. On an average number of drugs for each prescription were 3.16. Mean number of AMAs prescribed per condition was $0.9 \pm 0.05$ (mean \pm SEM). Monotherapy was advocated in $82.03 \% \%$ and polytherapy in $17.97 \%$ of patients.

Prescribing parameters obtained from this medical-audit study of RTIs illustrated that average number of drugs prescribed per prescription was 5.53. In a similar study conducted on URTIs in patients from ENT department the average number of drugs per prescription was $03 .{ }^{19}$ Similarly a study on pediatric patients showed the average drug per prescription to be $4.88 \pm 1.57 .{ }^{20}$

Two thirds $(67.6 \%)$ of the prescribed drugs were from the national list of essential medicines 2011 (NLEM). Percentage of drugs prescribed by generic name was negligible $(0.56 \%)$. Percentage of prescriptions with antibiotics was $52.68 \%$. Percentage of prescriptions with injection prescribed was $1.19 \%$. Percentage of prescriptions with antibiotics was $69 \%$. A study by Lalit Patil et al showed that the percentage of prescriptions with an antibiotic prescribed was $91.5 \%$ and the percentage of drug prescribed from essential drug list was $56.75 \%$. ${ }^{19}$ The drug utilization pattern followed in our hospital is in consensus with the standard guidelines. There is still scope for improvement with regard to use of drugs from Essential Drugs List.

\section{CONCLUSION}

This study essentially comprises a prospective medical audit study undertaken in a teaching hospital and attempts to describe the current prescription patterns in the treatment of RTIs. Data from a total of 603 medical case records pertaining to respiratory tract infective disorders 
were assessed. The prevalence of RTIs was slightly higher in males. The preferential AMAs employed were amoxicillin, amoxicillin-clavulinic acid combination, ceftriaxone, cefixime and azithromycin used individually. Of 12 different AMAs prescribed in this study, 6 drugs (penicillin, cephalosporin, aminoglycosodes, ATT, macrolides and fluroquinolones) falls within DU $90 \%$ segment. Bronchodilators and expectorants, mucolytics, cough suppressant, decongestants combination is noteworthy. The concurrent use of different groups of bronchodilators along with inhalational corticosteroids is also highlighted. Ranitidine, omeprazole and pantoprazole were the foremost gastroprotective agents. A variety of heterogenous adjuvants other than those mentioned above were administered and their roles in conferring additional therapeutic benefits have been emphasized. Majority of drugs used are from NLEM 2011. The overall impression about the prescription trends noted herein is suggestive of modest and rational approach in prescribing practices.

Funding: No funding sources

Conflict of interest: None declared

Ethical approval: The study was approved by the Institutional Ethics Committee

\section{REFERENCES}

1. Finch RG. Epidemiological features and chemotherapy of community-acquired respiratory tract infections. Journal of antimicrobial chemotherapy. 1990;26:53.

2. Nandimath MK, Ahuja S. Drug prescribing pattern in upper respiratory tract infection in children aged 1-14 years. International Journal of Pharma and Bio Sciences. 2012;3(1):299-308.

3. Jain N, Lodha R, Kabra SK. Upper respiratory tract infections. Indian J Pediatrics. 2001;68:1135-8.

4. Finegold SM, Johnson CC. Lower respiratory tract infection. The American Journal of Medicine. 1985;79(5):73-7.

5. Schaberg T, Torres A. Guidelines for management of adult community-acquired lower respiratory tract infections. Eur Respir J. 1998;11:986-91.

6. Guthrie R. Community-acquired lower respiratory tract infections: etiology and treatment. Chest. 2001;120(6):2021-34.

7. McCaig LF, Hughes JM. Trends in antimicrobial drug prescribing among office-based physicians in the United States. JAMA. 1995;273:214-9.

8. Health Canada and the Canadian Infectious Disease Society. Controlling antimicrobial resistance. An integrated action plan for Canadians. Can Commun Dis Rep. 1997;23:1-32.

9. Zhanel GG, Karlowsky JA, Palatnick L, Vercaigne L, Low DE, Hoban DJ. Prevalence of antimicrobial resistance in respiratory tract isolates of Streptococcus pneumoniae: results of a Canadian national surveillance study. The Canadian Respiratory Infection Study Group. Antimicrob Agents Chemother. 1999;43:2504-9.

10. Whitney CG, Farley MM, Hadler JH, Harrison LH, Lexau C, Reingold A, et al. Increasing prevalence of multi-drug resistant Streptococcus pneumoniae in the United States. N Engl J Med. 2000;343:1917-24.

11. Chen DK, McGeer A, De Azavedo JC, Low DE. Decreased susceptibility of Streptococcus pneumoniae to fluoroquinolones in Canada. $\mathrm{N}$ Engl $\mathrm{J}$ Med. 1999;341:233-9.

12. Davidson R, Cavalcanti R, Brunton JL, Bast DJ, De Azavedo JCS, Kibsey $\mathrm{P}$, et al. Resistance to levofloxacin and failure of treatment of pneumococcal pneumonia. N Engl J Med. 2002;346:747-50.

13. Prajapati V, Bhatt JD. Study of prescribing patterns of antimicrobial agents in the paediatric wards at tertiary teaching care hospital, Gujarat. IJPSR. 2012;3(7):2348-55.

14. Nelson CR. Drug utilization in office practice: National Ambulatory Medical Care Survey, 1990. Adv Data 1993;232:1-12.

15. Kumari IKS, Chandy SJ, Jeyaseelan L, Kumar R, Suresh S. Antimicrobial prescription patterns for common acute infections in some rural \& urban health facilities of India. Indian J Med Res. 2008;128:165-71.

16. Gaash B. Irrational Use of Antibiotics. Indian Journal for the Practicing Doctor. 2008;5(1):03-4.

17. Naik HG, Khanwelkar CC, Kolur A, Desai A, Gidamudi S. Drug Utilization Study on Antibiotics use in Lower Respiratory Tract Infection. National Journal of Medical Research. 2013;3(4):324-7.

18. Naik HG, Khanwelkar CC, Kolur A, Desai A, Gidamudi S. Drug Utilization Study on Antibiotics use in Upper Respiratory Tract Infection. International Journal of Recent Trends in Science and Technology. 2014;10(2):299-302.

19. Patil L, Khairnar A. Ninety percent drug utilization in patients of upper respiratory infections: Int. Res. J. Pharm. 2013;4(6):189-93.

20. Iyer GS, Patel PP, Panchal JR, Dikshit RK. An analysis of the pharmacological management of respiratory tract infections in pediatric in-patients at a tertiary care teaching hospital. International $\mathbf{J}$ of Medicine and Public Health. 2013;3(3):140-5.

Cite this article as: $\mathrm{Pal} \mathrm{P}$, Bhattacharyya $\mathrm{D}$, Kokila BN, Herle M, Ghosh A, Sen S. Drug utilization pattern in out-patients with respiratory tract infections in a rural teaching hospital: a prospective observational study. Int J Basic Clin Pharmacol 2017;6:2894-9. 